PROCEEDINGS OF THE

AMERICAN MATHEMATICAL SOCIETY

Volume 126, Number 5, May 1998, Pages 1321-1329

S 0002-9939(98)04231-2

\title{
WHEN MUST PURE EXTENSIONS OF COUNTABLE ABELIAN GROUPS NECESSARILY SPLIT?
}

\author{
H. PATRICK GOETERS AND PATRICK KEEF
}

(Communicated by Ronald M. Solomon)

\begin{abstract}
Necessary and sufficient conditions are given for the group of pure extensions of a countable abelian group by a countable abelian group to equal
\end{abstract} zero.

\section{INTRODUCTION}

In this work, by the term "group" we will mean "abelian group". Any notation and terminology not specifically defined can be found in [1].

If $A$ and $B$ are groups, then a short exact sequence $0 \rightarrow B \rightarrow X \rightarrow A \rightarrow 0$ is pure if for every integer $n, n B=B \cap n X$. The collection of pure extensions is denoted by $\operatorname{Pext}(A, B)$. This can be shown to agree with the first Ulm subgroup, $\operatorname{Ext}(A, B)^{1}=\bigcap n \operatorname{Ext}(A, B)$. Many different properties of this bifunctor are established in Chapter IX of [1], and we will assume the results contained there.

We will be interested in describing when $\operatorname{Pext}(A, B)=0$, where $A$ and $B$ are countable groups. This question was suggested to the first author by $\mathrm{C}$. Schochet, who noted its importance to subjects of $K K$-theory (see [2]). In particular, the graded Kasparov group $K K_{*}(M, N)$ of certain $C^{*}$-algebras $M$ and $N$ possesses a direct factor of the form $\operatorname{Pext}\left(K_{*}(M), K_{*}(N)\right)$. It is important to know when this latter group vanishes, and it should also be noted that every countable abelian group is $K_{0}(M)$ for some separable $C^{*}$-algebra $M$. In addition, as also noted in [2], by results of Jensen, if $M=\lim M_{i}$, then

$$
\operatorname{Pext}(M, N)=\varliminf^{1} \operatorname{Hom}\left(M_{i}, N\right)
$$

so that the collection of pure extensions is of some importance to topologists.

Our main result is Theorem 1 , where the above problem is completely solved. It is perhaps surprising that the question of when $\operatorname{Pext}(A, B)$ vanishes can be separated into two distinct cases; when $B$ is torsion and when it is torsion-free (Corollary $2)$. In addition, we show that whenever $A$ and $B$ are countable, then $\operatorname{Pext}(A, B)$ is either 0 or has cardinality $c$ (Corollary 3 ).

If $A$ is a group, then $t A$ will denote the torsion subgroup of $A$ and $f A \stackrel{\text { def }}{=} A / t A$. More generally, if $\mathcal{P}$ is a collection of primes, then $t_{\mathcal{P}} A$ will consist of those elements of $A$ whose order is a product of elements of $\mathcal{P}$, and $t_{\mathcal{P}}^{*} A$ will consist of those elements whose order is not divisible by any element of $\mathcal{P}$. We let $f_{\mathcal{P}} A=A / t_{\mathcal{P}} A$

Received by the editors October 30, 1996.

1991 Mathematics Subject Classification. Primary 20K20, 20K30, 16A65.

(c) 1998 American Mathematical Society 
and $f_{\mathcal{P}}^{*} A=A / t_{\mathcal{P}}^{*} A$. The integers localized at $\mathcal{P}$ will be denoted by $\mathbf{Z}_{(\mathcal{P})}$. A group $A$ will be said to be $\mathcal{P}$-free if $A_{(\mathcal{P})} \stackrel{\text { def }}{=} A \otimes \mathbf{Z}_{(\mathcal{P})}$ is a free $\mathbf{Z}_{(\mathcal{P})}$-module, and $\mathcal{P}$ - $\Sigma$-cyclic if $A_{(\mathcal{P})}$ is a direct sum of cyclic $\mathbf{Z}_{(\mathcal{P})}$-modules. If $\mathcal{P}=\{p\}$ is a single prime, we drop the parentheses in all of the above notation and terminology, e.g., $t_{p} A=t_{\{p\}} A$.

There are several standard identities and properties of localizations that we will utilize without comment. For example, if $A$ is a group, then there is a natural homomorphism $A \cong A \otimes \mathbf{Z} \rightarrow A_{(\mathcal{P})}$, whose kernel and cokernel are torsion groups with no $p$ torsion for any $p \in \mathcal{P}$. Another property which we use is the existence of a natural isomorphism $(f A)_{(\mathcal{P})} \cong f A_{(\mathcal{P})}$. Finally, if $\mathcal{P}$ and $\mathcal{Q}$ are collections of primes, $\mathcal{P} \cup \mathcal{Q}=\mathcal{R}$, and $A$ is a group with finite torsion-free rank, then $A$ is $\mathcal{R}$-free (or $\mathcal{R}$ - $\Sigma$-cyclic) iff it is both $\mathcal{P}$ - and $\mathcal{Q}$-free (respectively, $\mathcal{P}$ - and $\mathcal{Q}$ - $\Sigma$-cyclic).

The $\mathbf{Z}$-adic completion of a group $A$ will be denoted by $L A$, and if $p$ is a prime, the $p$-adic completion will be denoted by $L_{p} A$. There are natural homomorphisms $A \rightarrow L A, A \rightarrow L_{p} A$ and $L A \rightarrow L_{p} A$, which determine a natural isomorphism $L A \cong \prod_{p} L_{p} A$.

\section{Preliminaries}

If $A$ and $B$ are countable, and $0 \rightarrow Q \rightarrow P \rightarrow A \rightarrow 0$ is a free resolution of $A$, then there is a surjection $\operatorname{Hom}(Q, B) \rightarrow \operatorname{Ext}(A, B)$. Since $\operatorname{Hom}(Q, B)$ is the direct product of at most a countable number of copies of $B$, $\operatorname{Hom}(Q, B)$ has cardinality at most $c$, and so the same can be said of $\operatorname{Ext}(A, B)$. In many of our arguments, our fundamental strategy is to show that $\operatorname{Pext}(A, B)$ is non-zero by showing that its cardinality is at least $c$. This will, in turn, imply that its cardinality is exactly $c$. In other words, though we will primarily be concerned with when $\operatorname{Pext}(A, B)=0$, our arguments actually show that this group either vanishes or it has cardinality $c$.

Lemma 1. Suppose $A$ and $B$ are countable groups and $B$ is torsion-free. Let

$$
\mathcal{P}=\{p: p B \neq B\}
$$

Then $\operatorname{Pext}(A, B)=0$ iff $f A$ is $\mathcal{P}$-free.

Proof. If $X$ is a torsion group, then since $B$ is torsion-free, we have $\operatorname{Hom}(X, B)=0$. If, in addition, $X=t_{\mathcal{P}}^{*} X$, then $\operatorname{Ext}(X, B)$ is $p$-divisible for every $p \in \mathcal{P}$ (because multiplication by $p$ is an automorphism of $X$ ) and also $p$-divisible for every $p \notin \mathcal{P}$ (since multiplication by $p$ is an automorphism of $B$ ). This means that $\operatorname{Ext}(X, B)$ is divisible; but since $X$ is torsion, it must also be reduced (see 55.3 of [1]), proving that it is, in fact, zero.

The kernel and cokernel of the homomorphism $A \rightarrow A_{(\mathcal{P})}$ are torsion groups without $p$-torsion for any $p \in \mathcal{P}$. It follows from the last paragraph that the homomorphism $\operatorname{Ext}(A, B) \rightarrow \operatorname{Ext}\left(A_{(\mathcal{P})}, B\right)$ is an isomorphism, so that we may

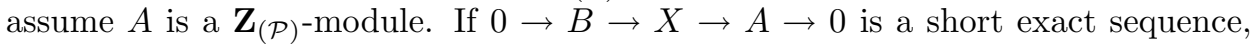
then since multiplication by any prime $q \notin \mathcal{P}$ is an automorphism of $A$ and $B$, it is also an automorphism of $X$. It follows that $X$ is also a $\mathbf{Z}_{(\mathcal{P})}$-module, so that the entire computation can be regarded as taking place over the $\operatorname{ring} \mathbf{Z}_{(\mathcal{P})}$.

For our next reduction, we begin with the observation that $\operatorname{Pext}(t A, B)=0$. To verify this, suppose $0 \rightarrow B \rightarrow X \rightarrow t A \rightarrow 0$ is a pure short-exact sequence. Purity implies that $t X$ maps onto $t A$, and since $B$ is torsion-free, this mapping is also injective, so that $t X \cong t A$ and the sequence splits. Considering the sequence

$$
\operatorname{Pext}(t A, B) \rightarrow \operatorname{Pext}(A, B) \rightarrow \operatorname{Pext}(f A, B) \rightarrow 0,
$$


it follows that $\operatorname{Pext}(A, B) \cong \operatorname{Pext}(f A, B)$, so that we may assume $A$ is torsion-free. This implies that $\operatorname{Ext}(A, B)$ is divisible, so that $\operatorname{Pext}(A, B)=\operatorname{Ext}(A, B)$.

To begin the proof, note that if $A$ is $\mathcal{P}$-free, then clearly $\operatorname{Pext}(A, B)=0$. Conversely, if $A$ is not $\mathcal{P}$-free, then by Pontryagin's Theorem, it has a finiterank pure submodule $A^{\prime}$ which fails to be $\mathcal{P}$-free. Since there is a surjection $\operatorname{Pext}(A, B) \rightarrow \operatorname{Pext}\left(A^{\prime}, B\right)$, if we can prove $\operatorname{Pext}\left(A^{\prime}, B\right) \neq 0$, the result follows. We may assume, therefore, that $A$ has finite torsion-free rank.

Let $F$ be a $\mathcal{P}$-free subgroup of $A$ of maximal rank. There is, therefore, an exact sequence

$$
\operatorname{Hom}(F, B) \rightarrow \operatorname{Ext}(A / F, B) \rightarrow \operatorname{Ext}(A, B)
$$

Observe $\operatorname{Hom}(F, B)$ is countable so the result will follow if we can show that $\operatorname{Ext}(A / F, B)$ is uncountable.

Since $A$ is not $\mathcal{P}$-free, $A / F$ must be infinite. There are two possibilities: either $A / F$ has a summand isomorphic to $\mathbf{Z}_{p \infty}$ for some $p \in \mathcal{P}$, or summands isomorphic to $\mathbf{Z}_{p^{k_{p}}}$ for an infinite set of primes $p \in \mathcal{P}$. In the first case, $\operatorname{Ext}(A / F, B)$ will have a summand isomorphic to $\operatorname{Ext}\left(\mathbf{Z}_{p^{\infty}}, B\right) \cong \operatorname{Hom}\left(\mathbf{Z}_{p^{\infty}}, \mathbf{Z}_{p^{\infty}} \otimes B\right)$ which is uncountable, and in the second, it will have a summand isomorphic to $\prod \operatorname{Ext}\left(\mathbf{Z}_{p^{k_{p}}}, B\right) \cong$ $\prod B / p^{k_{p}} B$, which is also uncountable. In either case, the result has been established.

Lemma 2. Suppose $A$ and $B$ are countable groups, $B$ is reduced and $p$ is a prime such that $t_{p} B$ is unbounded. If $\operatorname{Pext}(A, B)=0$, then $A$ is $p$ - $\Sigma$-cyclic.

Proof. Again, we prove that if $A$ is not $p$ - $\Sigma$-cyclic, then $\operatorname{Pext}(A, B)$ has cardinality at least $c$. By 56.1 of [1] there is a surjection

$$
\operatorname{Pext}(A, B) \rightarrow \operatorname{Pext}\left(A, B_{0}\right),
$$

so that if $\operatorname{Pext}\left(A, B_{0}\right)$ has cardinality $c$, then so does $\operatorname{Pext}(A, B)$. It follows that we may assume that $B^{1}=0$.

Since $0 \rightarrow t_{p}^{*} B \rightarrow B \rightarrow f_{p}^{*} B \rightarrow 0$ is pure, there is a surjection

$$
\operatorname{Pext}(A, B) \rightarrow \operatorname{Pext}\left(A, f_{p}^{*} B\right),
$$

so, once again, we may assume $B=f_{p}^{*} B$, i.e., $t_{p}^{*} B=0$.

By 57.4 of [1] there is a surjection

$$
\operatorname{Pext}(A, B) \rightarrow \operatorname{Hom}\left(A^{1}, L B / B\right) .
$$

Since $t_{p} B$ is countable and unbounded, it can be verified that $t_{p}(L B / B)$ is a divisible group of cardinality $c$. If $p^{\omega} t_{p} A \neq 0$, we could conclude that $\operatorname{Hom}\left(A^{1}, L B / B\right)$ has cardinality $c$, which implies that $\operatorname{Pext}(A, B)$ also has cardinality $c$. Therefore, we may assume $p^{\omega} t_{p} A=0$; since $A$ is countable, this implies that $t_{p} A$ is $\Sigma$-cyclic.

Since $A_{(p)}$ is not $\Sigma$-cyclic (as a $\mathbf{Z}_{(p)}$-module), we can conclude that $f A_{(p)}$ is not $\Sigma$-cyclic (since otherwise $A_{(p)} \cong f A_{(p)} \oplus t A_{(p)}$ would be $\Sigma$-cyclic). Hence there is a finite-rank pure subgroup $A^{\prime} \subseteq A$ containing $t A$ such that $A_{(p)}^{\prime}$ is not $\Sigma$-cyclic. There is a surjection $\operatorname{Pext}(A, B) \rightarrow \operatorname{Pext}\left(A^{\prime}, B\right)$, so we may assume $A=A^{\prime}$ has finite torsion-free rank.

We pause for one further reduction. Let $N=t_{p} A \oplus F$ be a $p$-basic subgroup of $A$ (i.e., $N$ is $p$-pure in $A$ and $A / N$ is $p$-divisible), where $F$ is a free subgroup of $N$. Note that $F$ will also be $p$-pure in $A$. We next let $M=\{a \in A: \exists m \in \mathbf{Z}, m a \in$ $F,(m, p)=1\}$, and $A^{\prime}=A / M$. We need to verify the following elementary facts: 
(1) $M / F$ is a torsion group with $t_{p}(M / F)=0$. This is obvious from the definition.

(2) $M$ is pure in $A$. If $a \in M$ and $m a \in F$ with $(m, p)=1$, then computing $p$-heights we have $h t_{M}(a)=h t_{M}(m a) \geq h t_{F}(m a)=h t_{A}(m a)=h t_{A}(a) \geq h t_{M}(a)$. If $q \neq p, a \in M$ and $a=q^{k} y$ for some $y \in A$, then $y \in M$, so that $q$-heights in $M$ and $A$ also agree.

(3) $t A^{\prime}=t_{p} A^{\prime} \cong t_{p} A$, under the natural map $A \rightarrow A^{\prime}$. If $a \in A$ and $n a \in M$, then $n=m p^{k}$, where $(m, p)=1$. It follows that $p^{k} a \in M$, so that $t A^{\prime}=t_{p} A^{\prime}$. To verify the last isomorphism, since $M$ is pure in $A, t A$ maps surjectively onto $t A^{\prime}$. Note that $t_{p} A \cap M=0$ and $t_{p}^{*} A \subseteq M$, so the isomorphism follows.

(4) $f A^{\prime}$ is $p$-divisible. This follows since $f A^{\prime}$ is an epimorphic image of $A / N$, which is $p$-divisible.

(5) $f A^{\prime} \neq 0$. If $f A^{\prime}=0$, then $A \cong M \oplus t_{p} A$, so that $A_{(p)} \cong M_{(p)} \oplus t_{p} A \cong$ $F_{(p)} \oplus t_{p} A$, which is a $\Sigma$-cyclic $\mathbf{Z}_{(p)}$-module.

The first fact implies $\operatorname{Hom}(M / F, B)=0$ so that there is an injection $\operatorname{Hom}(M, B)$ $\rightarrow \operatorname{Hom}(F, B)$. Since $F$ has finite rank and $B$ is countable, we can conclude $\operatorname{Hom}(F, B)$ is countable, which implies that $\operatorname{Hom}(M, B)$ is also countable. Considering the exact sequence

$$
\operatorname{Hom}(M, B) \rightarrow \operatorname{Pext}\left(A^{\prime}, B\right) \rightarrow \operatorname{Pext}(A, B),
$$

we can conclude that if $\operatorname{Pext}\left(A^{\prime}, B\right)$ has cardinality $c$, then so does $\operatorname{Pext}(A, B)$.

We therefore assume $A=A^{\prime}$, and that $x \in A$ has infinite order. It follows that if $n_{0}, n_{1}, n_{2}, \ldots$ is the $p$-height sequence of $x$, then either 1 ) there is a $j$ such that $n_{j} \geq \omega$, or 2 ) there are an infinite number of gaps (i.e., values of $i$ such that $\left.n_{i+1}>n_{i}+1\right)$.

Let $m_{0}, m_{1}, m_{2}, \ldots$ be a strictly increasing sequence of finite ordinals with an infinite number of gaps, such that $m_{i}<n_{i}$ for all but finitely many $i$.

Let $B=\left\{b_{1}, b_{2}, \ldots\right\}$ be an enumeration of $B$, and let $t_{p} B=\bigoplus_{u \in S} K_{u}$ where $S \subseteq \omega$ and for every $u \in S, K_{u} \neq 0$ is a direct sum of copies of $\mathbf{Z}_{p^{u}}$.

Since $B^{1}=0, B$ embeds as a pure subgroup of $L B \cong L_{p} B \oplus \prod_{q \neq p} L_{q} B$ with $L B / B$ divisible. We can think of elements of $L_{p} B$ as vectors with coordinates in a $p$-basic subgroup of $B$, which contains the terms $K_{u}, u \in S$.

Choose inductively a strictly increasing sequence $u_{i} \in S$, and elements $v_{i} \in K_{u_{i}}$, such that

1) $v_{i}$ generates a summand of $K_{u_{i}}$,

2) $m_{i}<u_{i}$

3) for every $j \leq i, b_{j}$ has $v_{i}$-coordinate divisible by $p^{m_{i}}$.

Let $\left\{R_{\alpha}: \alpha<c\right\}$ be an almost disjoint family of subsets of $S$. For each $\alpha<c$, let

$$
y_{\alpha}=\sum_{i \in R_{\alpha}} p^{m_{i}-i} v_{i} \in L_{p} B \subseteq L B
$$

[the fact that $y_{\alpha} \in L_{p} B$ follows from the fact that $m_{0}, m_{1}, m_{2}, \ldots$ has an infinite number of gaps].

Now, $L B / B$ is divisible and $x \in A$ has infinite order, so for every $\alpha<c$, there is a homomorphism

$$
f_{\alpha}: A \rightarrow L B / B
$$


such that $f_{\alpha}(x)=y_{\alpha}+B$. There is an exact sequence

$$
\operatorname{Hom}(A, L B) \rightarrow \operatorname{Hom}(A, L B / B) \rightarrow \operatorname{Pext}(A, B) .
$$

We claim that the $f_{\alpha}$ map to distinct elements of $\operatorname{Pext}(A, B)$, proving that this has cardinality $c$, as required. If this failed, then for some distinct $\alpha, \beta<c, f_{\alpha}-f_{\beta}$ would factor through a homomorphism $g: A \rightarrow L B$. Since $g(x)+B=f_{\alpha}(x)-f_{\beta}(x)=$ $y_{\alpha}-y_{\beta}+B$, we can find a $b_{j} \in B$ such that $g(x)=y_{\alpha}-y_{\beta}+b_{j}$. Choose $i \geq j$ such that $i \in R_{\alpha}-R_{\beta}$ and $m_{i}<n_{i}$. Then computing $p$-heights, we have

$$
n_{i}=h t\left(p^{i} x\right) \leq h t\left(p^{i} g(x)\right)=h t\left(p^{i} y_{\alpha}-p^{i} y_{\beta}+p^{i} b_{j}\right) .
$$

Notice that the $v_{i}$ th coordinate of $p^{i} b_{j}$ is divisible by $p^{m_{i}+i}>p^{m_{i}}, p^{i} y_{\beta}$ is 0 in this coordinate, and $p^{i} y_{\alpha}$ has $p^{m_{i}}$ in this coordinate. It follows that the $p$-height of $p^{i} y_{\alpha}-p^{i} y_{\beta}+p^{i} b_{j}$ is $m_{i}$, but this contradicts $m_{i}<n_{i}$.

If $p$ is a prime and $n \leq \omega$, then a subgroup $L$ of $A$ is $p^{n}$-pure if $p^{m} A \cap L=p^{m} L$ for every $m \leq n$. Note that if $n$ is finite and $L$ is torsion-free, then this reduces to requiring that $p^{n} A \cap L=p^{n} L$. We mention the following easy-to-check fact:

Lemma 3. Suppose $A$ is a group, $J, K$ are subgroups of $A, j$ and $k$ are integers with $j J \subseteq K$ and $k K \subseteq J, p$ is a prime not dividing $j, k$ and $n \leq \omega$. Then $J$ is $p^{n}$-pure iff $K$ is $p^{n}$-pure.

Let $\sigma=\left(n_{p}: p\right.$ is prime $)$ be a sequence of finite ordinals. A subgroup $L$ of $A$ will be called $\sigma$-pure if for each prime $p, L$ is $p^{n_{p}}$-pure in $A$. Similarly, $L$ is almost $\sigma$-pure if it is $p^{n_{p}}$-pure for all but finitely many primes $p$.

Corollary 1. Given the hypotheses of Lemma 3, $J$ is almost $\sigma$-pure iff $K$ is almost $\sigma$-pure.

\section{THE MAIN RESULT}

Theorem 1. Suppose $A$ and $B$ are countable groups and $B$ is reduced. Let

$$
\begin{aligned}
& \mathcal{P}=\{p: p f B \neq f B\}, \\
& \mathcal{Q}=\left\{p: t_{p} B \text { is unbounded }\right\} .
\end{aligned}
$$

In addition, let $\sigma=\left(n_{p}\right)$, where $n_{p}$ is the exponent of $t_{p} B$ if $t_{p} B$ is bounded and is 0 whenever $t_{p} B$ is unbounded. Then $\operatorname{Pext}(A, B)=0$ iff

(1) $f A$ is $\mathcal{P}$-free,

(2) $A$ is $\mathcal{Q}$ - $\Sigma$-cyclic, and

(3) every finitely generated subgroup of $A$ is almost $\sigma$-pure in $A$.

Proof. We begin by supposing that (1)-(3) are valid and show that $\operatorname{Pext}(A, B)=0$. By Lemma 1, (1) implies that $\operatorname{Pext}(A, f B)=0$. We now show that (2) and (3) imply that $\operatorname{Pext}(A, t B)=0$, which proves the result by considering

$$
\operatorname{Pext}(A, t B) \rightarrow \operatorname{Pext}(A, B) \rightarrow \operatorname{Pext}(A, f B) \rightarrow 0 .
$$

To this end, we may assume that $B$ is torsion. Since the kernel and cokernel of the natural map $A \rightarrow A_{(\mathcal{Q})}$ are torsion groups with no $q$-torsion for any $q \in$ $\mathcal{Q}$, (2) implies that $\operatorname{Pext}\left(A, t_{\mathcal{Q}} B\right) \cong \operatorname{Pext}\left(A_{(\mathcal{Q})}, t_{\mathcal{Q}} B\right)=0$, so we need to show $\operatorname{Pext}\left(A, t_{\mathcal{Q}}^{*} B\right)=0$. It follows that we may assume $B=t_{\mathcal{Q}}^{*} B$, i.e., that $B$ is torsion with bounded $p$-components. 
Let

$$
0 \longrightarrow B \longrightarrow X \stackrel{\pi}{\longrightarrow} A \longrightarrow 0
$$

be a pure sequence. Express $A$ as an ascending union $0=F_{0} \subseteq F_{1} \subseteq F_{2} \subseteq \ldots$ where each $F_{i}$ is finitely generated. Our objective is to define a splitting $\phi: A \rightarrow X$, defining it inductively, extending it from each $F_{i}$ to $F_{i+1}$. We will assume one particular property of such a partial splitting $\phi_{i}: F_{i} \rightarrow X$ : If $p$ is a prime, $m \leq n_{p}$ and $x \in F_{i} \cap p^{m} A$, then $\phi_{i}(x) \in p^{m} X$ (in other words, $\phi_{i}$ preserves $p$-heights up to $\left.n_{p}\right)$.

Since $F_{i+1}$ is almost $\sigma$-pure, there is a finite set of primes $\mathcal{T}$ such that for every $p \notin \mathcal{T}, F_{i+1}$ is $p^{n_{p}}$-pure in $A$. We can further expand $\mathcal{T}$ by adding a finite collection of primes in order to guarantee that $t_{\mathcal{T}}^{*}\left(F_{i+1} / F_{i}\right)=0$. Let $B_{0}=t_{\mathcal{T}} B$ and $B_{1}=t_{\mathcal{T}}^{*} B$, so that $B=B_{0} \oplus B_{1}$. Note that $B_{0}$ is bounded, so that $X=B_{0} \oplus X_{1}$, and there is a pure sequence

$$
0 \longrightarrow B_{1} \longrightarrow X_{1} \stackrel{\pi}{\longrightarrow} A \longrightarrow 0
$$

Let $\rho: X \rightarrow B_{0}$ and $\mu: X \rightarrow X_{1}$ be the usual projections. Note that $\mu \circ \phi_{i}: F_{i} \rightarrow$ $X_{1}$ is a partial splitting of $\pi$. Since $F_{i+1}$ is finitely generated, by purity there is a partial splitting $\eta: F_{i+1} \rightarrow X_{1}$. It should be observed that we are not assuming $\eta$ extends $\phi_{i}$ or $\mu \circ \phi_{i}$.

There is an exact sequence

$$
\operatorname{Hom}\left(F_{i+1}, B_{1}\right) \rightarrow \operatorname{Hom}\left(F_{i}, B_{1}\right) \rightarrow \operatorname{Ext}\left(F_{i+1} / F_{i}, B_{1}\right)
$$

and since $B_{1}=t_{\mathcal{T}}^{*} B_{1}$ and $t_{\mathcal{T}}^{*}\left(F_{i+1} / F_{i}\right)=0$, the right group vanishes. Next, observe that $\mu \circ \phi_{i}-\left.\eta\right|_{F_{i}}$ is a homomorphism $F_{i} \rightarrow B_{1}$, so that $\mu \circ \phi_{i}-\left.\eta\right|_{F_{i}}=\left.\lambda\right|_{F_{i}}$ for some homomorphism $\lambda: F_{i+1} \rightarrow B_{1}$.

The last paragraph shows that $\mu \circ \phi_{i}$ extends to a partial splitting $\gamma=\eta+\lambda$ : $F_{i+1} \rightarrow X_{1}$. Note that if $p \notin \mathcal{T}, m \leq n_{p}$, and $x \in p^{m} A \cap F_{i+1}$, then by $p^{n_{p}}$-purity, $x \in p^{m} F_{i+1}$, so that $\gamma(x) \in p^{m} X_{1}$, and hence $\gamma$ preserves $p$-heights up to $n_{p}$ for all $p \notin \mathcal{T}$.

Next, consider $\kappa=\rho \circ \phi_{i}: F_{i} \rightarrow B_{0}$. Let $n=\prod_{p \in \mathcal{T}} p^{n_{p}}$, so that $n B_{0}=0$. Note $\kappa$ induces a homomorphism $\bar{\kappa}: F_{i} /\left(F_{i} \cap n A\right) \rightarrow B_{0}$ and that $\bar{\kappa}$ does not decrease $p$-heights (calculated in $A / n A$ ) for any $p \in \mathcal{T}$. Therefore, $\bar{\kappa}$ extends to a homomorphism $A / n A \rightarrow B_{0}$. Let $\kappa^{\prime}$ be the composition $F_{i+1} \rightarrow A \rightarrow A / n A \rightarrow B_{0}$, so that $\kappa^{\prime}$ preserves $p$-height up to $n_{p}$ for each $p \in \mathcal{T}$.

We can now extend $\phi_{i}$ to $\phi_{i+1}: F_{i+1} \rightarrow X$ by setting it equal to $\left(\kappa^{\prime}, \gamma\right): F_{i+1} \rightarrow$ $B_{0} \oplus X_{1}$. This is clearly a splitting; to show that it preserves heights, let $p$ be a prime and $x \in F_{i+1}$. If $p \notin \mathcal{T}$, then $\kappa^{\prime}(x)$ has infinite $p$-height, so that the $p$ height of $\phi_{i+1}(x)$ equals the $p$-height of $\gamma(x)$ which is at least the $p$-height of $x \in A$ (whenever this is at most $n_{p}$ ). Similarly, if $p \in \mathcal{T}$, then the $p$-height of $\phi_{i+1}(x)$ equals the $p$-height of $\kappa^{\prime}(x)$ which is at least as large as the $p$-height of $x \in A$. Therefore, one direction of the proof is complete.

We now show that if $(1),(2)$ or $(3)$ fails, then $\operatorname{Pext}(A, B) \neq 0$. Suppose first that (1) fails. Lemma 1 implies that $\operatorname{Pext}(A, f B) \neq 0$, and since there is a surjection

$$
\operatorname{Pext}(A, B) \rightarrow \operatorname{Pext}(A, f B) \rightarrow 0
$$

we can conclude $\operatorname{Pext}(A, B) \neq 0$.

We next assume (3) fails. Suppose $F$ is a finitely generated subgroup of $A$ which is not almost $\sigma$-pure. Let $k$ be an integer such that $k F$ is free. By Corollary $1, k F$ also fails to be almost $\sigma$-pure, so there is no loss of generality in assuming that $F$ 
is free. Now, let $\mathcal{S}$ be an infinite set of primes such that $p^{n_{p}} A \cap F \neq p^{n_{p}} F$ for all $p \in \mathcal{S}$.

Let $A^{\prime}$ be the pure hull of $F$ (i.e., $A^{\prime} / F=t(A / F)$ ). Since $A^{\prime}$ is pure in $A$, for each $p \in \mathcal{S}, F$ is not $p^{n_{p}}$-pure in $A^{\prime}$. There is a surjection

$$
\operatorname{Pext}(A, B) \rightarrow \operatorname{Pext}\left(A^{\prime}, B\right) \rightarrow 0,
$$

so if we can show $\operatorname{Pext}\left(A^{\prime}, B\right) \neq 0$, we will be able to conclude $\operatorname{Pext}(A, B) \neq 0$, as required. We may therefore assume $A=A^{\prime}$ has finite rank and $A / F$ is torsion.

Claim: $\operatorname{Pext}(A, t B)$ has cardinality $c$.

Before continuing, we note how this implies $\operatorname{Pext}(A, B) \neq 0$. There is an exact sequence

$$
\operatorname{Hom}(A, f B) \rightarrow \operatorname{Pext}(A, t B) \rightarrow \operatorname{Pext}(A, B) .
$$

Note that $\operatorname{Hom}(A, f B) \cong \operatorname{Hom}(f A, f B)$, and since $f A$ has finite rank and $f B$ has countable rank, this group is countable, and the claim follows.

Therefore, we may assume $B=t B$ is torsion. For each $p \in \mathcal{S}$, let $\left\langle r_{p}\right\rangle$ be a summand of $t_{p} B$ of exponent $n_{p}$. If $B^{\prime}=\bigoplus_{p \in \mathcal{S}}\left\langle r_{p}\right\rangle$ and $\operatorname{Pext}\left(A, B^{\prime}\right)$ has cardinality $c$, then it follows that $\operatorname{Pext}(A, B)$ also has cardinality $c$, as required. So we may assume $B=B^{\prime}$.

For each $p \in \mathcal{S}$, let $m_{p} \leq n_{p}$ be minimal such that $p^{m_{p}} A \cap F \neq p^{m_{p}} F$, and let $x_{p} \in\left(p^{m_{p}} A \cap F\right)-p^{m_{p}} F$. The minimality of $m_{p}$ implies that $x_{p}$ has $p$-height $m_{p}-1$ in $F$ (since otherwise $x_{p} \in\left(p^{m_{p}-1} A \cap F\right)-p^{m_{p}-1} F$ ). Let $y_{p} \in F$ satisfy $p^{m_{p}-1} y_{p}=x_{p}$. Now choose $z_{p} \in A$ such that $p^{m_{p}} z_{p}=x_{p}$. The minimality of $m_{p}$ also implies that $p^{m_{p}-1} z_{p} \notin F$ (since otherwise $p^{m_{p}-1} z_{p} \in\left(p^{m_{p}-1} A \cap F\right)-p^{m_{p}-1} F$ ). Let $A^{\prime}$ be the subgroup of $A$ generated by $F$ together with each $z_{p}(p \in \mathcal{S})$. There is a surjection

$$
\operatorname{Pext}(A, B) \rightarrow \operatorname{Pext}\left(A^{\prime}, B\right) \rightarrow 0
$$

[to verify this, if $0 \rightarrow Q \rightarrow P \stackrel{\rho}{\rightarrow} A \rightarrow 0$ is a pure sequence with $P$ and $Q \Sigma$-cyclic and $P^{\prime}=\pi^{-1}\left(A^{\prime}\right)$, then there are homomorphisms $\operatorname{Hom}(Q, B) \rightarrow \operatorname{Pext}(A, B) \rightarrow$ $\operatorname{Pext}\left(A^{\prime}, B\right)$ whose composite is a surjection]. If we can show the latter group has cardinality $c$, it also follows that the first group has that cardinality. So, without loss of generality, we assume $A=A^{\prime}$.

Let $\left\{\mathcal{S}_{\alpha}\right\}_{\alpha<c}$ be a pairwise almost disjoint family of infinite subsets of $\mathcal{S}$. For each $\alpha<c$, let $X_{\alpha}$ be generated by

(1.1) $F$,

(1.2) $r_{p}, z_{p}$, for $p \notin \mathcal{S}_{\alpha}$,

(1.3) $u_{p}, v_{p}$ for $p \in \mathcal{S}_{\alpha}$,

subject to the relations,

(2.1) $p^{n_{p}} r_{p}=0, p^{m_{p}} z_{p}=p^{m_{p}-1} y_{p} \in F$, for $p \notin \mathcal{S}_{\alpha}$,

(2.2) $p^{n_{p}} u_{p}=p^{n_{p}} y_{p} \in F, p^{m_{p}} v_{p}=p^{m_{p}-1} u_{p}$, for $p \in \mathcal{S}_{\alpha}$.

These generators and relators determine an extension

$$
E_{\alpha}: 0 \longrightarrow B \stackrel{\lambda_{\alpha}}{\longrightarrow} X_{\alpha} \stackrel{\pi_{\alpha}}{\longrightarrow} A \longrightarrow 0
$$

by assigning for each $p \in \mathcal{S}_{\alpha}$,

(3.1) $\lambda_{\alpha}\left(r_{p}\right)=u_{p}-y_{p}$,

(3.2) $\pi_{\alpha}\left(u_{p}\right)=y_{p}$

(3.3) $\pi_{\alpha}\left(v_{p}\right)=z_{p}$. 
The sequence $E_{\alpha}$ is pure; in fact, for every prime $p, 0 \rightarrow t_{p} B \rightarrow t_{p} X_{\alpha} \rightarrow t_{p} A \rightarrow 0$ actually splits. To see this, note that if $p \notin \mathcal{S}_{\alpha}$, then $t_{p} X_{\alpha}=\left\langle r_{p}\right\rangle \oplus\left\langle p z_{p}-y_{p}\right\rangle$, and if $p \in \mathcal{S}_{\alpha}$, then $t_{p} X_{\alpha}=\left\langle u_{p}-y_{p}\right\rangle \oplus\left\langle p v_{p}-u_{p}\right\rangle$. In either case, the first summand corresponds to $t_{p} B$ and the second to $t_{p} A$.

Let $p \in \mathcal{S}$. We claim that $F$ is $p^{n_{p}}$-pure in $X_{\alpha}$ iff $p \in \mathcal{S}_{\alpha}$. To verify this, note that if $p \notin \mathcal{S}_{\alpha}$, then $p^{m_{p}-1} y_{p} \in\left(p^{m_{p}} X_{\alpha} \cap F\right)-p^{m_{p}} F$, so that $F$ is not $p^{n_{p}}$-pure in $X_{\alpha}$. If $p \in \mathcal{S}_{\alpha}$, then $F / p^{n_{p}} F$ can be seen to embed naturally as a summand of $X_{\alpha} / p^{n_{p}} X_{\alpha}$, and the claim follows.

We now claim that for distinct $\alpha, \beta<c, E_{\alpha}$ is not equivalent to $E_{\beta}$, proving that $\operatorname{Pext}(A, B)$ has the required cardinality. In fact, we show that $X_{\alpha}$ and $X_{\beta}$ are not isomorphic. To show this is the case, we assume that they are and derive a contradiction. Given such an isomorphism, we can identify $X_{\alpha}$ and $X_{\beta}$, giving a single group $X$. Note that $F$ embeds in both $X_{\alpha}$ and $X_{\beta}$, but after this identification we may get two distinct subgroups which we denote by $F_{\alpha}$ and $F_{\beta}$, respectively. There are integers $j$ and $k$ such that $j F_{\alpha} \subseteq F_{\beta}, k F_{\beta} \subseteq F_{\alpha}$. Since $\mathcal{S}_{\alpha}-\mathcal{S}_{\beta}$ is infinite, there is a prime $p \in \mathcal{S}_{\alpha}-\mathcal{S}_{\beta}$ which does not divide $j, k$. This means that $F_{\alpha}$ is $p^{n_{p}}$-pure in $A$, while $F_{\beta}$ fails to be $p^{n_{p}}$-pure in $A$, contradicting Lemma 3.

We now suppose that it is (2) that fails. If for some $p \in \mathcal{Q}, A_{(p)}$ fails to be a $\Sigma$ cyclic $\mathbf{Z}_{(p)}$-module, then by Lemma 2 we can conclude $\operatorname{Pext}(A, B) \neq 0$, so we may assume each such $A_{(p)}$ is $\Sigma$-cyclic. In particular, we may assume $t_{\mathcal{Q}} A=\bigoplus_{p \in \mathcal{Q}} t_{p} A$ is $\mathcal{Q}$ - $\Sigma$-cyclic.

There is a sequence

$$
0 \rightarrow t_{\mathcal{Q}} A \rightarrow A_{(\mathcal{Q})} \rightarrow(f A)_{(\mathcal{Q})} \rightarrow 0,
$$

so we may assume $f A$ is not $\mathcal{Q}$-free. This implies that there is a pure subgroup $A^{\prime} \subseteq A$ of finite torsion-free rank such that $f A^{\prime}$ is not $\mathcal{Q}$-free. Since there is a surjection

$$
\operatorname{Pext}(A, B) \rightarrow \operatorname{Pext}\left(A^{\prime}, B\right) \rightarrow 0,
$$

if we can show $\operatorname{Pext}\left(A^{\prime}, B\right) \neq 0$, then $\operatorname{Pext}(A, B) \neq 0$, as required. It suffices, then, to assume that $A=A^{\prime}$ has finite torsion-free rank.

Let $F$ be a free subgroup of $A$ of maximal rank, let $\mathcal{R}$ be the set of primes $p \in \mathcal{Q}$ such that $F$ is $p^{\omega}$-pure in $A$, and let $\mathcal{S}=\mathcal{Q}-\mathcal{R}$ be the remaining ones. Note that $F_{(\mathcal{R})}$ will be a pure $\mathbf{Z}_{(\mathcal{R})}$-submodule of $A_{(\mathcal{R})}$. Since $A_{(\mathcal{R})} / F_{(\mathcal{R})}$ is torsion, it follows that $A_{(\mathcal{R})}$ splits into $t_{\mathcal{R}} A \oplus F_{(\mathcal{R})}$, so that $(f A)_{(\mathcal{R})}$ is $\mathcal{R}$-free. Now for each $p \in \mathcal{S}$ we already know $(f A)_{(p)}$ is $p$-free, so that if $\mathcal{S}$ is finite, we can conclude $(f A)_{(\mathcal{Q})}$ is $\mathcal{Q}$-free, contrary to assumption.

It follows that $\mathcal{S}$ is finite and for each $p \in \mathcal{S}$ there exists a positive integer $m_{p}$ for which $F$ fails to be $p^{m_{p}}$-pure in $A$. For each $p \in \mathcal{S}$, let $t_{p} B=K_{p} \oplus L_{p}$ where $K_{p}$ is a bounded subgroup of exponent $n_{p} \geq m_{p}$. If $B^{\prime}=B /\left(\bigoplus_{p \in \mathcal{S}} L_{p}\right)$, then $t_{\mathcal{S}} B^{\prime} \cong \bigoplus_{p \in \mathcal{S}} K_{p}$, so that for this $A$ and $B^{\prime}$, condition (3) fails. By what we've proven already, this implies that $\operatorname{Pext}\left(A, B^{\prime}\right) \neq 0$, and since there is a surjection

$$
\operatorname{Pext}(A, B) \rightarrow \operatorname{Pext}\left(A, B^{\prime}\right) \rightarrow 0,
$$

we conclude that $\operatorname{Pext}(A, B) \neq 0$, as required.

We now point out two consequences of the above proof:

Corollary 2. If $A$ and $B$ are countable groups, then $\operatorname{Pext}(A, B)=0$ iff $\operatorname{Pext}(A, f B)$ $=\operatorname{Pext}(A, t B)=0$. 
Proof. We may clearly assume $B$ is reduced. Essentially condition (1) is equivalent to $\operatorname{Pext}(A, f B)=0$, and conditions (2) and (3) are equivalent to $\operatorname{Pext}(A, t B)=0$.

Corollary 3. If $A$ and $B$ are countable groups, then $\operatorname{Pext}(A, B)$ is either 0 or it has cardinality $c$.

\section{REFERENCES}

1. L. Fuchs, Abelian Groups, vol. 1, Academic Press, New York. 1970. MR 41:333

2. C. Schochet, The UCT, the Milnor sequence, and a canonical decomposition of the Kasparov groups, $K$-Theory 10 (1996), 49-72. MR 97d:46088

Department of Mathematics, Auburn University, Auburn, Alabama 36849

Department of Mathematics, Whitman College, Walla Walla, Washington 99362 